\title{
Micromole per 24 Hours
}

National Cancer Institute

\section{Source}

National Cancer Institute. Micromole per 24 Hours. NCI Thesaurus. Code C67406.

A unit of amount of substance flow rate equivalent to the rate at which one millionth of a mole of substance crosses a given surface or is delivered to a given object or space over a period of time equal to 24 hours. 